\title{
High-Performance SAR Image Formation and Post-Processing
}

\author{
E. Appiani, M. Corvi, G. Garibotto, C.Coelho \\ Elsag Bailey, Research \& Development Services, \\ v. Puccini 2, 16154 Genova, Italy \\ Email: \{eappiani, mcorvi, ggaribotto,coelho\}@elsag.it
}

\begin{abstract}
This paper presents two experiences in the field of high-performance SAR processing. Getting high quality SAR images in reasonably short times is getting more and more strategic for remote sensing applications, still requiring the use of data-parallel processing. Fast formation of images from radar data requires dedicated machines suited to fine-grained, DSP-based parallelism, while improving the image quality through despeckling is suited to large-grained parallelism based on general-purpose machines. These two aspects are analyzed and evaluated by presenting: an industrial system for real-time SAR processing on a custom, DSP-based parallel system, called ACIP-XSAR; and the results of the Esprit PULSAR project, providing a portable and scalable parallel package for image despeckling and segmentation.
\end{abstract}

\section{Introduction}

SAR imaging is a highly computationally intensive application field, both for the SAR image formation and for any post processing aimed at improving or analyzing the images. The SAR image formation involves a double integration of the raw data, known as range and azimuth compressions, aimed to invert the physical process of SAR imaging. This computation has an essentially fine grained structure, provided that the FFT process is separable in the two directions.

On the other hand, the SAR image postprocessing attempts to improve the quality of the whole images, strongly affected by multiplicative noise, the so-called speckle noise, intrinsecally inherent to the imaging process. The post processing is important to enhance the images and to make them more useful for exploitation, mostly for environmental monitoring. It consists in "global" operations that must involve regions of pixels at the same time, therefore requiring a large grained computing structure.

These two aspects of the SAR image processing has being studied and employed at ElsagBailey, which developed an industrial product for real-time image formation from X-band data, named ACIP-XSAR, on the other hand being involved in the Europort-2 project for parallel industrial applications within the PULSAR consortium, which studied a parallelization of postprocessing routines for despeckling and segmentation of SAR images [1].

The project partners of PULSAR, finished at the end of 1995, were:

- NASoftware, Liverpool, UK, as code provider;

- Liverpool University, Liverpool, UK, as parallel expert;

- Elsag-Bailey, Genoa, IT, as industrial partner and parallel expert;

- Scot Conseil, Toulouse, FR, as application expert.

PULSAR is a parallel, portable and scalable package for SAR post-processing, documented with benchmarking reports on some commercial high performance platforms, a quality evaluation and a classification of possible applications [6]. The rest of the paper focusses on the different computing features and parallel solutions for SAR image formation and despeckling, the first being suited to a small grained parallelism (high data throughput, low intensivity), while the latter being suited to a large grained one (low data throughput, high intensivity) . 


\section{Industrial Real-time SAR Processing}

The advantages of SAR processing instead of optical acquisition for remote sensing are well known, and will not be illustrated in this paper; [8] is a basic text on the SAR theory and systems. In the SAR field, "real-time" has not the usual correct meaning of servicing asynchronous events with firm deadlines, but it indicates the ability to form SAR images at the same pace, or at least similar, as the raw data were collected.

This ability allows to observe earth zones not too much later than data acquisition, for instance in order to get quick decisions in case of disasters (especially pollution) or to correct the antenna position and working parameters on line. Radar data are not processed directly at their reception from the antenna, but stored in high-capacity, high-speed tapes, for re-using the data, filtering the transmission noise and pre-summing them for reducing their volume.

Despite the rapid progress in computer technology, real-time SAR still requires highperformance architectures, being in the range of GFLOPS, also depending on the image quality and resolution. SAR formation being mostly based on FFTs, fine-grained MIMD machines including DSPs are useful to process a sustained, seamless input data flow. For the range compression, each line of data is sent to a different DSP for being processed independently of the others and being recollected after the processing. For the azimuth compression, data blocks are transposed before and after the processing, which is carried out in the same way.

The Elsag Bailey most recent SAR system, called ACIP-XSAR (advanced image processor for X-band SAR) is made of three DSP-based multiprocessing nodes called EMMA2E. This is the last generation of custom parallel machines for image and signal processing, also used in OCR and pattern recognition applications [2].

\subsection{The EMMA2E Architecture}

EMMA2E is a NUMA (non-uniform memory access) shared-memory multiprocessor aimed to best exploit "local" accesses from processors and DSPs to their own memories. The global shared address space is useful to achieve fast data communication between processors, while local subspaces allows for fast computation without suffering the delay for memory contention: a compromise between distributed and shared memory machines.

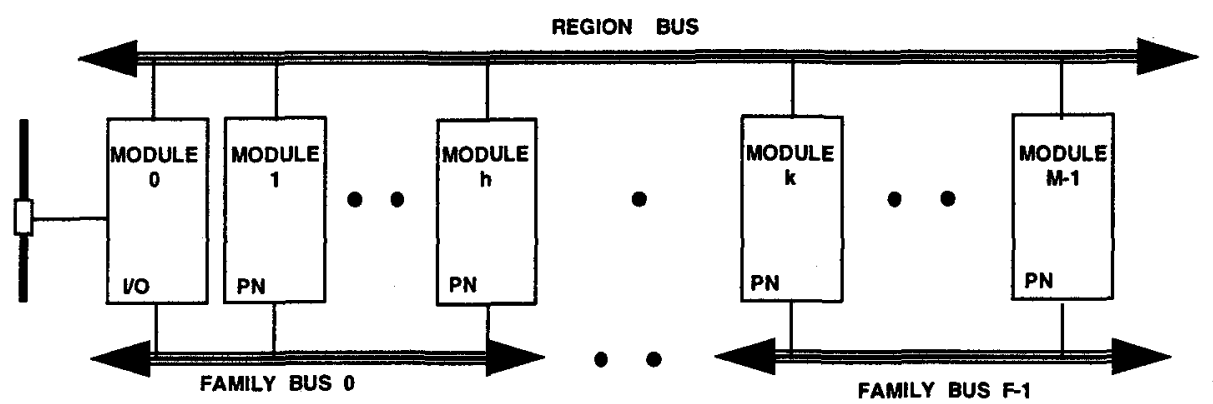

EXAMPLE SCHEME OF AN EMMA2E TARGET NODE WITH

- ONE VO MODULE FOR LAN INTERFACE

- M-1 PN MODULES, FOR A TOTAL OF M MODULES AND $4 M-3$ PROCESSORS

- F FAMILY BUSSES

Fig. 1. The EMMA2E architecture 
The interconnection is based on double hierarchy of linear busses with 64-bit data path and 32-bit address path. Processing modules may be: I/O front-ends, handling standard or custom peripherals with one processor each; and computing modules (called PN) with four processor each. Any processor is based on a 486 running at $33 \mathrm{MHz}$, possibly coupled with a DSP 96001 as slave coprocessor for intensive floating point computation, sharing the same address space as the main processor (fig. 1).

The maximum configuration consists of a LAN interface module, needed for communication and supervision from a standard host node, and $20 \mathrm{PNs}$ with 80 processor-coprocessor sets, with a total peak computation rate of about 1000 MIPS +2000 MFLOPS. The peak point-to-point rate in a bus is $64 \mathrm{MB} / \mathrm{s}$ (Mega Bytes per second, as we will indicate in the rest of the paper), when moving data between shared memories with custom programmable DMA controllers. The total rate depends on the number of secondary busses in a configuration; in the most typical cases there are one primary bus and three secondary ones, providing a peak rate of $256 \mathrm{MB} / \mathrm{s}$.

\subsection{The ACIP-XSAR System}

ACIP-XSAR relies on a three-stage pipeline of EMMA2E parallel nodes (fig. 2):

- the first devoted to range compression;

- the second to the azimuth compression, and

- the last to deskewing the images, according to the computed antenna position and motion parameters.

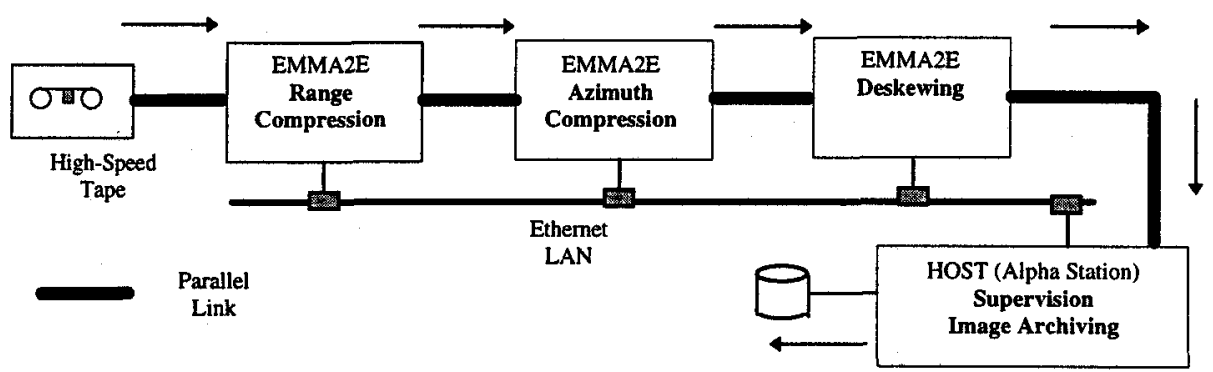

Fig. 2. ACIP-XSAR distributed configuration

This functional pipeline, starting with a high-speed tape device in input, is connected through:

- point-to-point fast channels ( $32 \mathrm{MB} / \mathrm{s}$ ) for the data flow, and

- a standard LAN for supervision and control,

this last in charge to a standard host which also performs image visualization and archiving. When forming low resolution, quick look images, data flow rates are: $8 \mathrm{MB} / \mathrm{s}$, on input to the range and azimuth compression nodes (complex data with 16-bit ranges); and $4 \mathrm{MB} / \mathrm{s}$ on input to the deskew and supervision nodes (real data). The total sustained computation rate is 860 MFLOPS; the three machines, including 90 NS96000 DSPs, could reach three times that performance, but the overall process is conditioned by computation/communication rate and the low disk output rate of the supervision node.

The ACIP-XSAR is in use at the ASI (Italian Space Agency) premises in Matera. This configuration was employed in two missions of the NASA Space Shuttle including experiments of SAR acquisition, in April and August 1994. Each missions lasted about one week and ACIPXSAR was able to provide Earth images day-by-day, a few hours after their acquisition, in time to evaluate their quality and give feedback to the Shuttle crew. 


\section{PULSAR: High-Performance SAR Post-Processing}

To increase the applicability of SAR images it is necessary to improve their quality and reduce the speckle noise [9]. The PULSAR code provider, NASofware, developed SAR despleckling and segmenting algorithms based on a repeated non-linear transformation of the pixel values, of which the most important is called Anneal [10] [11], a simulated annealing algorithm. The image pixel values are estimated trying to fit an a-priori pixel distribution in the neighborhood of every pixel itself. The execution depends of the number of iterations of the annealing procedure and of the look number, which defines the expected a-priori pixel statistics. The look number is related to the actual look number used in the formation of the SAR image.

Due to the high intensivity on the data and to the large size of typical SAR images (usually 8000 pixels per side), it is generally difficult, or even impossible, to apply sequentially these algorithms to SAR images. It is therefore important to develop a parallel approach to the SAR image post-processing, with the double objective of reducing the response time and relaxing the memory requirements over multiple nodes.

The PULSAR project adopted a distributed, data-parallel solution well suited for large-grain computation. The proposed solution upon a distributed heterogeneous platform environment uses a message passing library, PVM or MPI, and an upper layer for the data-parallel organization. This is enforced by the Distributed Data Library (DDL), developed by the University of Liverpool and based on the Single Program Multiple Data model (SPDM) [4]. The application code is the same for all the machines involved in the computation. DDL implicitly splits any image into a mono- or bi-dimensional grid of subimages, one per process, according to the number of processes involved.

Elsag Bailey mainly contributed to the parallel benchmarking [7] and to the quality evaluation of Anneal [9], besides the validation of the code portability and the development of a set of parallel support routines [3]. Furthermore, we have been involved in the Anneal test and benchmarking on a full size SAR image.

\section{PULSAR Benchmarking}

The PULSAR codes were considered for benchmarking on high-performance platforms and a quality and performance evaluation of Anneal was carried out. The purpose of this benchmark was to demonstrate that the parallelization of the algorithm did not introduce artifacts (detectable at a visual inspection) related to the splitting of the images among the tasks, while showing that Anneal has acceptable speedup and scalability [7].

The computing platforms adopted for benchmarking were a cluster of 8 DEC Alpha stations at Bergen, Norway, a 16-node Meiko CS-2 at CERN, Geneva, and a 48 node GC PowerPLus at Padeborn, Germany.

Three test images were ERS-1 images selected for their relevance to environmental resource monitoring, with 16-bits pixel values, $12.5 \mathrm{~m}$ range and azimuth resolution, obtained with 3 nonoverlapping looks of $320 \mathrm{~Hz}$ each. They represent two land scenes with fields and crops (agricultural monitoring) and a sea scene with oil spills (environment pollution control). The mentioned applications are good candidates to benefit from PULSAR and the quality evaluation has been carried upon them [9].

The benchmarking was aimed at evaluating both the parallel speedup (by increasing parallelism with the same problem size) and scalability (by increasing parallelism proportionally to the problem size). Both turned out to be quite good in the range from 1 to 16 processes on all the machines. Input data were square portions of the test images with 512, 1024, 2048 and 4096 pixels per side, with sizes ranging from $1 \mathrm{MB}$ to $64 \mathrm{MB}$ (megabytes). The tests consisted of runs with different problem sizes on different numbers of computing nodes. Due to the wide range of 


\section{ANNEAL Scaling on CS-2}

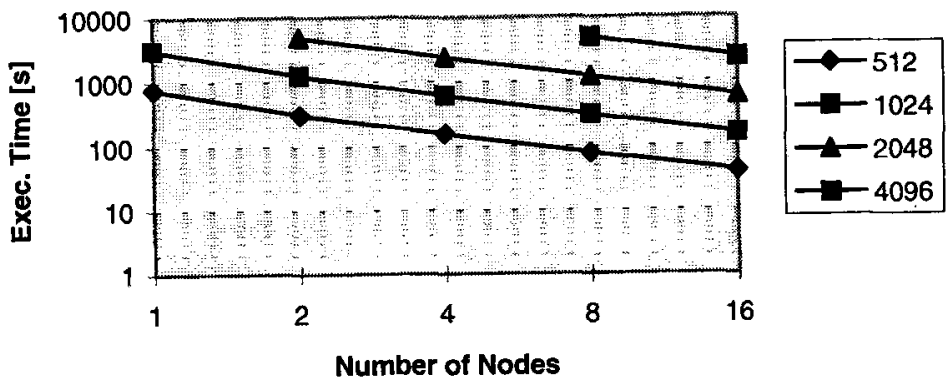

Fig. 3. Anneal performance for different square image sizes (pixels per side)

sizes, and hence of memory and computation requirements, it was sensible to apply parallel subranges proportionally to each problem size.

The quality assessment of the parallel executions was carried out with the use of the support routines. These allowed to do pixelwise comparisons on images coming from parallel and sequential processing, as well as on images processed with different kinds of parallelization. The accuracy tests showed that the pixel-by-pixel values' differences between the parallel and sequential annealed images were predominantly smaller than 25 , quite low with respect to the full dynamics of 0 - 65535. Unfortunately, these are statistic tests which do not take into account possible peculiar pixel roles in the visual appearance of images, but are the simplest way to characterize the relative weights of the differences between similar images.

The scalability was measured in the same way as for the speedup, by comparing the execution times of tests in which the degree of parallelism was proportional to the problem size. To illustrate both scalability and speedup features, fig. 5 shows the Anneal computing times on the CS-2 as a function of the number of nodes and for different image sizes, expressed as pixels per side. When doubling the image side length, the problem size scales by 4 , and we get similar execution times by using 4 times the number of nodes, thus proving the linear performance scaling. These results suggest that normalized times, with respect to the number of nodes and the number of image pixels, can be used to obtain an adequate, although far from perfect, estimate of performance. Anneal takes about 2.4 ( $\mathrm{sec}$ node)/Kpixel on the CS-2, 1.5 on the Alpha, and 2.2 on the GC (for 50 iterations).

\section{Anneal Quality Evaluation}

Two kinds of targets have been considered for the quality evaluation: point targets and distributed targets. The quality indices for point targets are the spatial resolution, the integrated side-bole ratio (ISLR) and the peak sidelobe ratio (PSLR), while the one for the distributed targets is the radiometric resolution.

The spatial resolution is a measure of the capability of the SAR instrument to distinguish between two adjacent point targets. We consider the spatial resolution both in range and in azimuth. The one dimensional spatial resolution is defined as the width of a point target response function where the intensity is $3 \mathrm{~dB}$ below the peak value. The side-lobe ratios are a measure of the capability of the SAR instrument to identify weak targets surrounded by bright targets area. The ISLR is defined as the ratio of the emergy in the sidelobes to that in the mainlobe. 
The mainlobe is the rectangle whose sides are twice the spatial resolutions on the two coordinates, centered at the peak. The sidelobe region is the remaining area within a rectangle of side twenty times the spatial resolutions, centered at the peak. The PSLR is defined as the ratio of the peak intensity in the sidelobe region to that of the peak in the mainlobe. A good despeckler should not reduce the resolution of the image, so that the postprocessed image would not appear blurred but have sharp edges. This, in fact, should distinguish a simple low-band pass filter from the PULSAR complex and intensive algorithms. The radiometric resolution represents the capability of the SAR system to distinguish between neighboring radiometric levels. It measures the despeckling power, i.e., how well the algorithm can remove the noise from the image. It is defined as $\gamma=10 \log (1+\sigma / \mu) \quad$ where $\mu$ and $\sigma$ are the mean and standard deviation of the distributed target region statistics. A good despeckler should improve the radiometric resolution on uniform areas, i.e. reduce its numerical value.

The Anneal processing has two input parameters: the look number and the iteration number. We have measured the quality indices as function of each of these two parameters, while keeping the other fixed. As test images we employed 512x512 subimages of the three ERS1 PULSAR benchmark images, and two X-SAR images, of the same size. The latter are 16-bit images with $3.5 \mathrm{~m}$ range and azymuth resolution and an azymuth bandwidth of $1000 \mathrm{~Hz}$ (1 look), representing urban scenes with clutters of buildings and streets. Due to the improved resolution and to the urban environment represented, X-SAR images proved to be even more suited than ERS-1 ones to the qualitative and quantitative evaluaton of despeckling [9], whose results are not presented in details in this paper.

Point target measurements were carried out on the two land scene ERS-1 images. The results show that the point target resolution is only slightly decreased (numerically increased), usually about $10 \%$, while the sidelobe Ratios are improved, passing from $-4.2 \mathrm{~dB}$ to about -2 $\mathrm{dB}$. The radiometric resolution shows a definite improvement passing from 1.2 to about 0.5 . It increases slowly with the look number, and decreases (improves) with the iteration number. For the other images the results have analogous behaviour, although the numerical values may be somewhat different. Similar conclusions were obtained on the X-SAR images.

\section{Anneal Benchmark with a Full-Size SAR Image}

An Anneal benchmark on a full size image was carried out on the Meiko CS-2 cluster at CERN, using all the 16 nodes available ( 2.4 GFLOPS peak performance). The $8200 \times 8000$ ERS1 image, used as test case by the ASI (Italian Space Agency), is about 260MB and Anneal requires $1 \mathrm{~GB}$ of memory for the processing.

The Anneal run was performed with 20 iterations and look number 6 . It took about 5000 seconds, of which 4200 for the actual computation, confirming the scaling projections computed with smaller images. Figure 6 shows a $512 \times 512$ portion of the original and the despeckled images, whose dynamics has been cut for visualization. In fact, due to the corner reflectors, the original image dynamics range is from 11 to 65535 , but the average level is just 300 and the standard deviation 197. The despeckled image statistics reflects a range compression, from 25 to 60412 , with average 289 and standard deviation 157, this last much reduced for the effect of noise removal. The peak value of the point target is reduced at $2 / 3$ of the original value, but without affecting the resolution significantly: the original resolution is $1.96 * 1.49$ pixels, while the annealed one is $2.06 * 1.57$. 


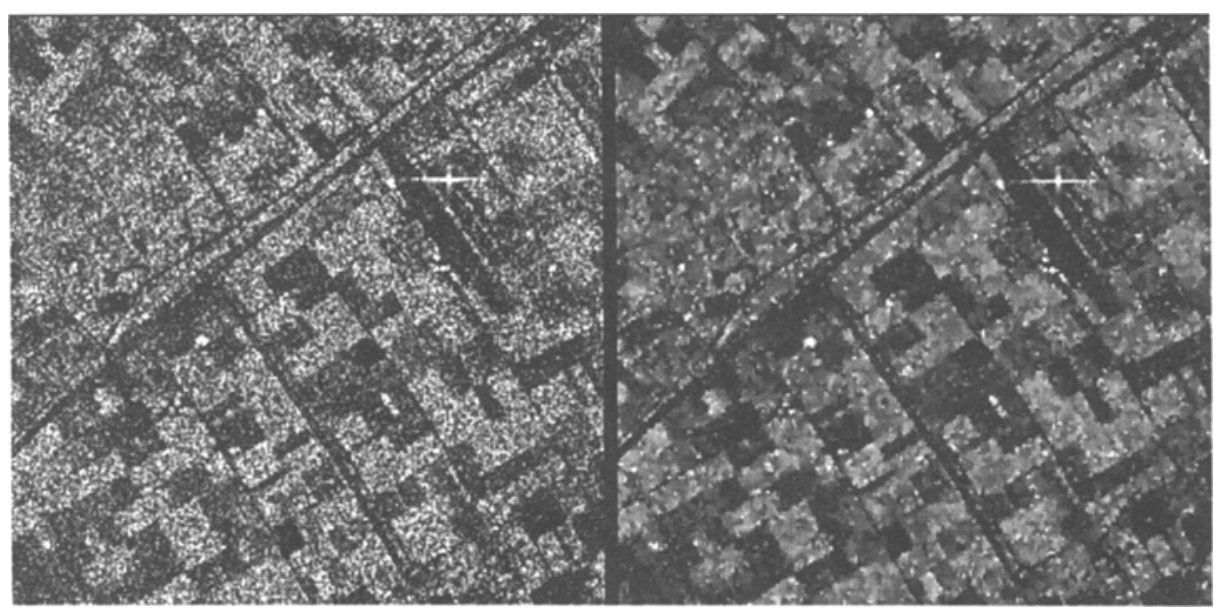

Fig. 4. A SAR image of Holland (left) and the same despeckled by Anneal (right)

\section{Conclusions}

The paper presented two fields of activities related to SAR processing: the image formation, suited to fine-grained parallelism and DSP-based architectures, and the image post-processing for despeckling, suited to large-grained parallelism. The PULSAR project, presented in its most relevant results, showed that despeckling codes have good speedup and scalability, due to their high computation to communication ratio.

This scaling capability on platforms with reasonable cost promises a way to achieve well balanced computation times for SAR image formation and despeckling, thus being able to offer products integrating the two kinds of platforms (dedicated with small grained parallelism, general purpose with large grained parallelism) and related functionalities. A further technological factor of convergence is brought by the more and more powerful superscalar RISCs, also including image processing operators in their instruction set (like the new Sun UltraSparc): they will probably greatly reduce the need for DSP-based architectures even for real-time SAR processing, thanks also to the high-speed internal buses and large memories.

\section{Acknowledgement}

The authors wish to thank the partners of Europort PULSAR consortium, and in particular M.Delves, R.Cook, C.Addison and J. Harms, for their fruitful contribution and invaluable support in the development of the project.

\section{References}

1. ESPRIT EUROPORT2 TECHNICAL ANNEX: PART H, "PARALLEL UNIVERSAL LIBRARY FOR SAR", Smith Engineering, Sep 1993

2. E. Appiani, "EMMA2E EROS: INTRODUCTION AND GENERALITY", Elsag Bailey doc. n. RJ4.9034.132.60, Nov 1993

3. M. Corvi et al., "SUPPORT ROUTINES: USER GUIDE", PULSAR working paper 3.2-1, Elsag Bailey, June 1995

4. C. Addison et al., "DISTRIBUTED DATA REQUIREMENTS IN PULSAR", PULSAR wokring paper 2.2-2, Univ. of Liverpool, Dec. 1994 
5. Geist, Beguelin, Dongarra, Jiang, Manchek, Sunderam, "PVM3 USER'S GUIDE AND REFERENCE MANUAL", ORNL, Oak Ridge, TN 37831, USA, May 1993

6. J. Harms et al., "PULSAR EVALUATION FINAL REPORT", PULSAR working paper 5.3-1, Scot Conseil, Dec 1995

7. C. Addison et al., "BENCHMARK RESULTS", PULSAR working paper 5.3-2, Univ. of Liverpool, Dec 1995

8. Curlander, R.N. Mc Donough, "SYNTHETIC APERTURE RADAR: SYSTEMS AND SIGNAL PROCESSING", J. Wiley \& Sons, 1991

9. Frost et al., "A MODEL FOR RADAR IMAGES AND ITS APPLICATION TO ADAPTIVE DIGITAL FILTERING OF MULTIPLICATIVE NOISE", IEEE Trans. on PAMI, vol 4, p. 157, 1982

10.NASoftware Ltd., "CAESAR USER GUIDE v1.1", Defence Research Agency, UK, 1994

11.R.G. White, "A SIMULATED ANNEALING ALGORITHM FOR RADAR CROSS-SECTION ESTIMATION AND SEGMENTATION", SPIE Intern. Conf. on Applications of Artificial Neural Networks, 1994 